\title{
A Lightweight App Distribution Strategy to Generate Interest in Complex Commercial Apps: Case Study of an Automated Wound Measurement System
}

\author{
Min Tang \\ Arizona State University \\ Mesa, AZ USA \\ mtang16@asu.edu
}

\author{
Kevin A. Gary \\ Arizona State University \\ Mesa, AZ USA \\ kgary@asu.edu
}

\author{
Özgür Güler \\ eKare Inc. \\ Fairfax, VA USA \\ oguler@ekareinc.com
}

\author{
Patrick Cheng \\ eKare Inc. \\ Fairfax, VA USA \\ pcheng@ekareinc.com
}

\begin{abstract}
Tablet-based healthcare technologies automating clinical triage procedures hold exciting promise for increased precision and expediency. These point-ofcare (POC) solutions are often complex, and their introduction to the marketplace may encounter cost and usability barriers. One example triage procedure is wound measurement. This paper demonstrates an innovative approach to POC wound measurement by introducing a free "light" version of a wound measurement mobile app that serves as a teaser for a full-featured commercial offering. We first describe the commercial offering; a 3D wound assessment tablet application. Then we present the smartphone app that inherits features from the tablet app. The smartphone app adopts a simple scaling algorithm to address the lack of a highly advanced computer vision system for the automated wound measurement task that exists in the tablet app. This paper describes the design process for developing this smartphone app, provides a detailed exposition of the scaling algorithm, and discusses the significance of this approach to app development and distribution.
\end{abstract}

\section{Introduction}

Wound assessment is a very important part of wound care in the modern medical industry. Correct diagnosis and effective documentation through assessment is essential to treat wounds effectively. A meta-analysis by Jorgensen et al. [1] of conventional two-dimension (2D) wound assessment measures and various three-dimension (3D) measures concluded that 2D measures (specifically digital planimetry and digital imaging) are the most accurate and reliable. Another recent meta-analysis by Khoo and Jansen [2] also concludes that digital planimetry is a reliable method. Both studies acknowledge the lack of a gold standard in the field. These traditional approaches are invasive, inaccurate, inconsistent and time-consuming, with extra training needed for the nurses and caregivers who carry out the measurement $[3,4]$. It is essential that nurses have adequate proficiency in wound measuring to assess wounds correctly. However, new 3D measures have been postulated as being able to improve accuracy and eventually economic viability. Shah, Wollak, and Shah [5] recently suggested 3D scanning as an improvement over 2D methods.

The required steps of wound measuring include manual measurement, taking the wound picture, and data transfer (shown in Figure 1). The entire process may take more than two and a half minutes, and yield imprecise measurements.

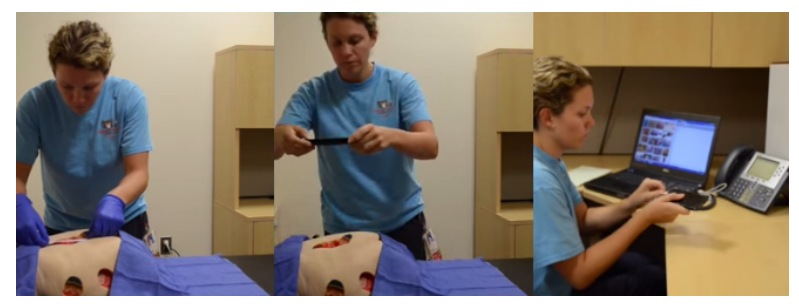

Figure 1. Traditional wound measurement

eKare Inc., a health provider from the metro Washington D.C. area, has come up with a disruptive solution to revolutionize the wound assessment process. eKare successfully developed a tablet based 3D wound assessment application that combines the most advanced mobile computing, 3D sensing and cloud computing technologies in a single system. The entire wound measurement process is simplified by taking a photo of the wound, and having the software automatically analyze the picture to generate wound related data,. This data is then synchronized to eKare's HIPAA compliant cloud database.

Though eKare's 3D wound assessment application is immensely powerful, currently it only runs on an iOS tablet (iPad) outfitted with a special camera attachment, and it is not free. To facilitate market adoption, a lightweight smartphone app (mHealth app) was created that could approximate the results and impact on clinical workflow processes in wound measurement. By creating a free alternative that demonstrated the efficiency of automated wound measurement, the hope was for greater market penetration and upsell opportunities. Through a collaborative effort between eKare and the Health Engineering Applications Laboratory (HEAL) at 
Arizona State University (ASU), a smartphone mHealth POC app was designed and developed that approximates eKare's powerful 3D wound assessment app. This app is referred to as a "wallet app", in that it emphasizes the form factor; an iPhone can be carried in the pocket for convenience and ready application. This new wallet app is designed for the iPhone, it is portable, easy to use, and completely free.

\section{Background}

Several efforts have been made to improve wound measurement accuracy while at the same time shorten the lengthy process and simplify the entire procedure. Digital and portable technologies are the leading trends in the market, and various devices, applications and methodologies have been created to realize these goals.

\subsection{Competing Vendors and Research Projects}

Visitrak [6] is a popular product that has been on the market for almost a decade. This product comprises a digital measuring tablet with stylus, a 3-layer tracing grid and foam tipped depth indicator. To use Visitrak to perform 2D wound area measurement, the user places a grid over the wound area, and then uses a permanent marker pen to trace around the edge of the wound. Finally, the user places the tracing grid on the surface of the Visitrack digital tablet, and uses the stylus to trace the wound outline on the grid until the tablet processes the outline and stores it as a digital copy. For wound depth measurement, an extra step is performed by sticking the depth indicator into the deepest part of the wound to read in depth data. Though this product does digitize the wound measurement result and improves the precision of the measurement, it follows the same path as conventional methods when measuring wound areas because the whole process is still carried out manually (using marker pen to draw the outline instead of using paper tape) and depth measurement is done basically in the same way as conventional methods. Currently the dominant portion of the market utilizes the similar technologies to handle wound measurement [7-12].

\subsection{Commercial Offering from eKare}

eKare developed a tablet-based system to automate the entire wound assessment process. A user only needs to take a photo of the wound area, and enter some basic information like the wound location and wound type.

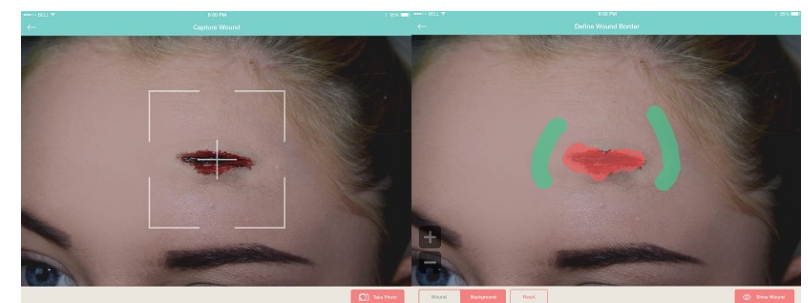

Figure 2. Capture wound photo by CompX app

After the wound image is captured, the user defines (roughly) the inside and the outside of the wound with finger swipes, as shown red and green in Figure 2, right respectively. The interactive segmentation algorithm delineates the wound border using the user annotation automatically and obtains 3D wound dimensions (area and depth) and tissue classification through highly intelligent computer vision algorithms. Figure 2 shows the wound scan results for a wound assessment. The wound information is obtained instantaneously and integrates the wound image seamlessly so that the user can obtain a full set of wound measurements.

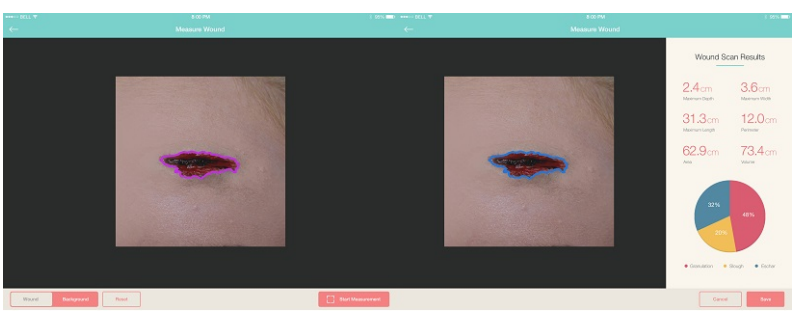

Figure 3. CompX 3D wound analysis

To maximize usability and ensure seamless integration into the clinical flow, eKare provides a cloud management platform, an Amazon Web Services (AWS) cloud database, which supports the front-end device. Once a wound photo is taken and analyzed, the photo and wound data generated are automatically uploaded to the cloud database. Using the HIPAAcompliant platform, clinicians can easily and securely archive, access, and analyze the wound images and informatics generated from the eKare sensor.

\section{Creating the wallet app}

As described in Section 1, the objective of this project was to produce a "wallet app" suitable for low barrier entry into the market. For this approach to be successful, the wallet app had to replicate the essential features of the commercial offering without access to the 3D vision features and proprietary algorithms. To accomplish this, the focus was on creating a design that captured the effective disruption of the clinical 
workflow in the same way the commercial app does to reduce barriers to commercial adoption. To replace the proprietary commercial features the team implemented an accepted 2D method base don digital planimetry and validated in a recent clinical study [13]. This section describes the requirements, design, and implementation methods used to create the wallet app.

\subsection{Requirements and Design Process}

The requirements engineering approach was based on C-PLAD [14]. This approach recognizes reusable application components through scenario-driven requirements analysis. In this application, the task was to identify what components from the tablet app could be reused and which needed to be varied, in a productline sense, in the smartphone app. The design approach followed that of [15], where prototypes were used to identify key protocol (process steps) needed in the app.

The requirements engineering process began by conducting a review of eKare's documentation and videos of the existing project. A meeting was held to validate the team's understanding of the current system architecture and workflow of the commercial tablet app. eKare provided a report defining the goals of the wallet app and the deliverable (transition) requirements. The team responded by producing prototypes to reify the functionality desired in the new wallet app (example prototype shown in Figure 4).

Wound Info

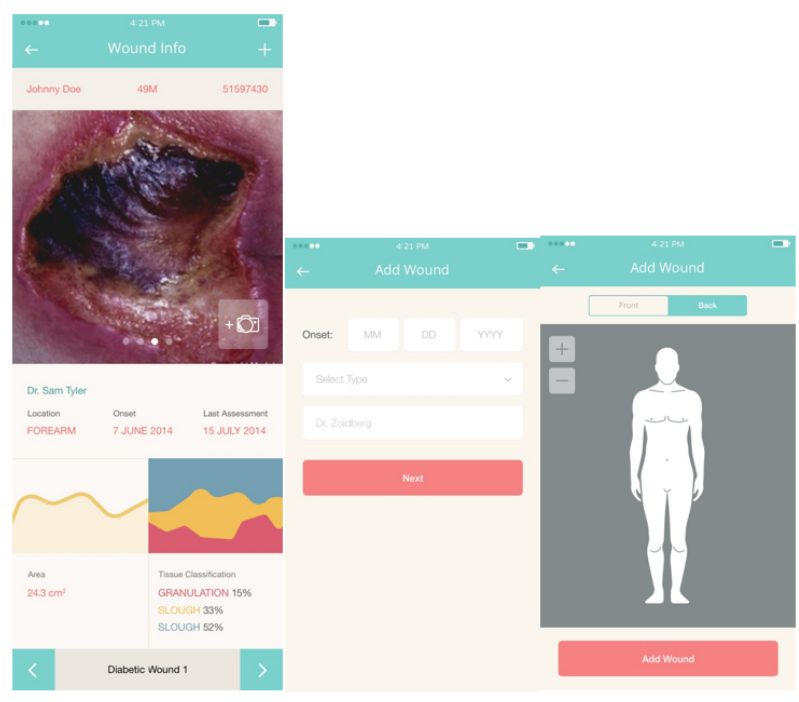

Figure 4. Prototype wound measurement page

The prototypes were reviewed for general usability characteristics and consistent theming and branding. More importantly, the prototypes allowed the team to conduct a gap analysis to ensure the wallet app would remain faithful to the same clinical workflow as the commercial application to the greatest extent possible. This was critically important as preserving the integrity of the clinical workflow created a streamlined path for user adoption of the commercial version, creating upsell market opportunities. In the Xcode platform, the storyboard tool was used to layout the transitions between screens representing different steps of the clinical workflow. A panoramic view showing the transitions is given in Figure 5; the details of each particular screen are not as important as the flow.

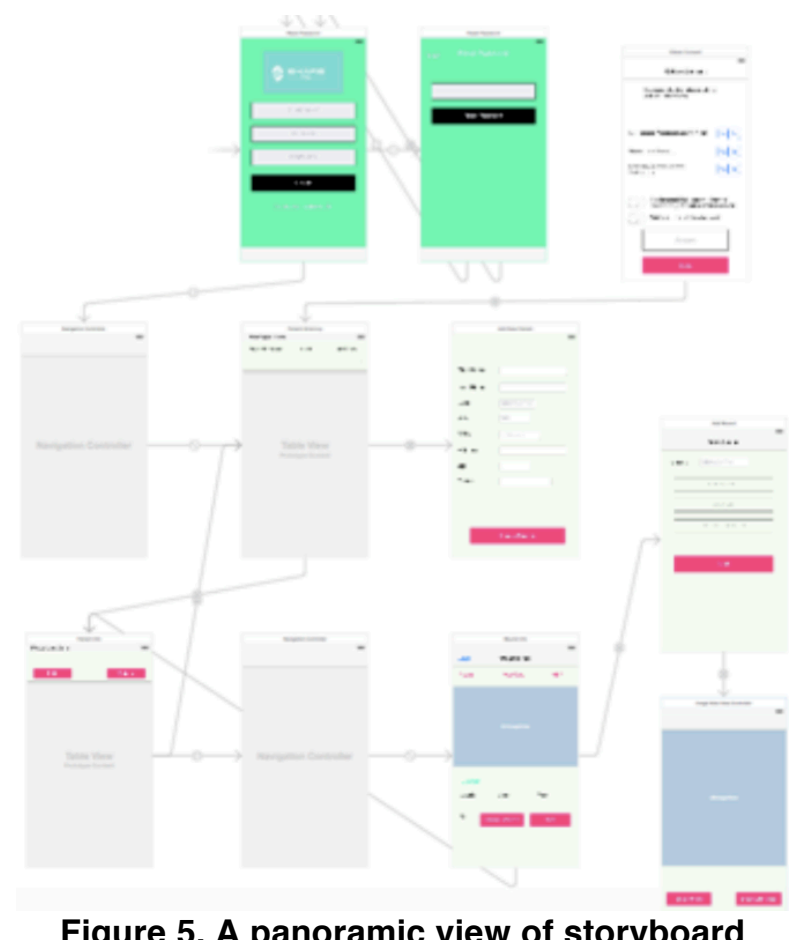

Figure 5. A panoramic view of storyboard

Finally, a usability evaluation and comparison to the commercial application was conducted and resulted in some small design revisions including:

- Modifying for the location and appearance of the top-level search bar on the Patient Directory feature;

- Modify data presentation alignment on the same feature;

- Location of Edit and Delete buttons on the Patient Info screen;

- Change in widget UI selection on the Obtain Consent screen (see Figure 6 left);

- On the same Obtain Consent screen use a textbox instead of a digital signature feature.

- The removal of some advanced information such as tissue classification and auto-generated graphs on the Wound Info screen (see Figure 6 right), and a front/back segmentaton control on the picturetaking screen due to $2 \mathrm{D}$ limitations. 
These modifications are largely cosmetic and user experience driven; they do not significantly alter the clinical workflow enabled by the technology.
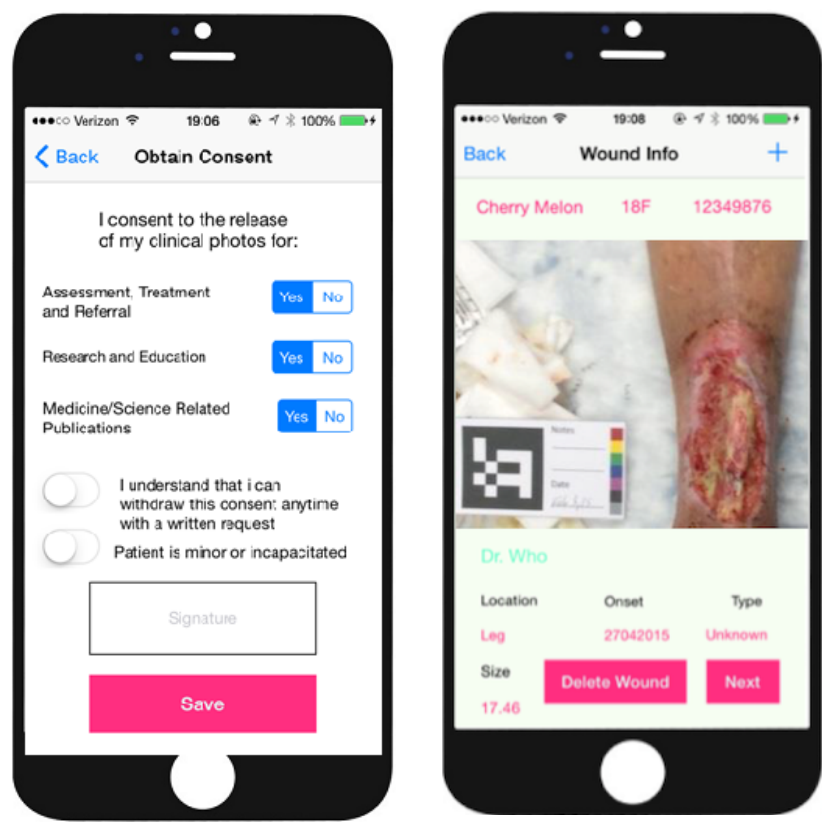

Figure 6. Obtain Consent, Wound Info screens

\subsection{Implementation Phase}

In a combined effort of eKare and the HEAL of ASU, this project focused on building a lightweight version of eKare wound assessment app. The iPhone has been selected as the device that runs this app. By "lighweight", we mean the smartphone app does not fully inherit the sophisticated computer vision algorithm which is the core technology in the commercial version. Instead, the wallet app utilizes digital planimetry, a widely-used 2D scaling algorithm, to compute the size of the wound using a reference maker (shown in Figure 4).

The data storage component of the wallet app was changed from cloud-based to local to the mobile device. Hence the wallet app stores all patient related data (including wound images) locally in a SQLite database instead of synchronizing with the cloud.

Most other features from the commercial version are preserved in this wallet app including user authentication, patient record management, patient consent acquirement, and wound photo capture and management. This is critically important as an upselling aspect of the free wallet app concept. It allows end users to understand the impact of a disruptive technology on their clinical workflow, while enticing them into purchasing the commercial version with its more powerful vision algorithms.
During this phase the design elements related to usability and flow described in the preceding section were implemented; this section focuses on a few key features related to capturing images and scaling (digital planimetry) wound measurements from those images. The section concludes with some specific details on the code-level implementation relevant to these features.

\subsubsection{Saving and loading pictures}

Implementing the camera feature was not difficult, but having the app save the image properly and reload it later was more challenging. This issue is also related to the design of database. Currently two tables are created in the database, one for patient general information while the other is mainly for the wound records. It is impractical to save an image file directly in the database, instead the most viable solution is to save the location where the image is stored in the database. Multiple wound records should be able to coexist and relate to the same patient. Under this assumption, the second table needs to be changed because there cannot be multiple entries in the table with the same primary key (patient's MRN). It is only natural to make the image file name or file path to be the primary key because this information must be unique. The file name of the wound picture is selected as the primary key because all wound pictures are stored in the same location (the document folder) as it is unique on the device.

\subsubsection{Scaling Method Design}

The commercial tablet's app "killer feature" is the ability to automate wound measurement using proprietary intelligent computer vision algorithms. The wallet app does not have the system level environment (3D camera, screen form factor size, processing capability) to replicate this behavior on the smartphone. Therefore digital planimetry, a 2D alternative measurement process, was designed and implemented for the wallet app (Section 3). The digital planimetry design utilizing and external reference marker and finger taps measurement (Figure 2) was the biggest technical challenge of the wallet app development. The basic concept is as follows:

1. When taking the wound picture, hold a reference object (in this case a rectangle reference card with known dimensions is used) right next to the wound (can be placed up, down, left, or right as long as at the same distance with the wound) and make sure both wound and reference object are captured within the boundary of the photo (see Figure 7).

2. Now the size of the reference object is known prior to the measurement, let the width be $\mathrm{Wr}$ and height 
be $H r$ in metric coordinates (these can be measured using a ruler). The desired result is the size of the wound $S w$ with width $W w$ and height $H w$. Since it is a simplified means to measure the approximate size of a wound with irregular shape, it is assumed that the wound resides in an invisible "rectangular box" which contains the wound, and the size of that "box" is calculated and treated as the approximate size of the wound.

3. After the wound picture is taken, the user can start measuring the size of the wound (the "rectangular box") and the reference card in the picture. Because both objects are of rectangular shape, a total of 8 points needed to be marked down for both objects (4 points for each): one point at the left edge and one point at the right edge and the horizontal distance between these two points equal to the width of the object. The same goes for the points at the top edge and the bottom edge to calculate the height. After all 8 points are marked down, we have width and height of the wound $W p w$ and $H p w$, and width and height of the reference card $\mathrm{Wpr}$ and $\mathrm{Hpr}$ in pixel coordinates. Because the sizes of the wound and reference object shown in the picture are simply scaled down from their real sizes, the real size of the wound can be calculated as:

$$
\begin{gathered}
\text { eq1. } W w / W r=W p w / W p r=>W w=(W r * W p w) / W p r \\
H w / H r=H p w / H p r=>H w=(H r * H p w) / H p r \\
S w=W w * H w
\end{gathered}
$$

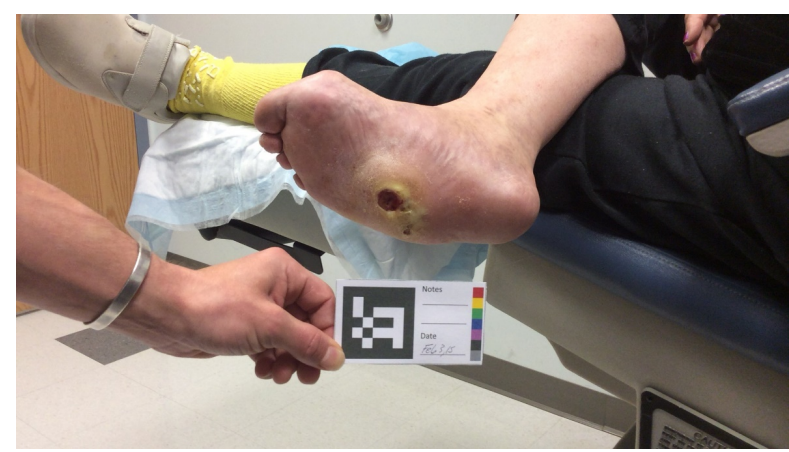

Figure 7. Digital planimetry $w /$ reference marker

\subsubsection{Implementation Details}

After a wound picture is taken and chosen by the user, it will be displayed in a UIImageView on the Take Wound Picture page. By default UIImageView disables the user interaction, so the user interaction setting has to be set to "Yes" before the user can mark down the points. This change of setting would take effect immediately after user clicks "Measure Size" button. In order to help the user better keep track of the marked points, a special drawing function is utilized to draw a small red round dot where the user touches the screen. The red dots will not disappear until the measurement is done so the user doesn't have to remember all the previous marked points, and this approach also visually improves the measurement procedures. One thing that needs to be noted is that the Multitouch feature is not enabled under this circumstance, so the user has to mark down the points sequentially not simultaneously. The order of marking points has to start with the wound, and then the reference card.

Marked points are stored as CGPoint, which is defined by CGGeometry Reference as one of the Application Services Frameworks of iOS. CGPoint simply models a point in a 2D dimension which has $x$ and $y$ coordinates. The $x, y$ coordinates are represented using CGFloat datatype defined in iOS. The distance between two CGPoint A and B is calculated using the following formula:

$$
\begin{aligned}
& \text { eq2.HorizDist }=A \cdot x-B . x \\
& \text { VertDist }=A . y-B . y \\
& \operatorname{dist}(A, B)=\sqrt{\text { HorizDist } 2+\text { VertDist } 2}
\end{aligned}
$$

This process is repeated until all required widths and heights are obtained. Then calculate the size of the wound using formulas listed in section 5.1. One thing may easily be overlooked is that CGPoint is using Pixel as its unit, which is different from the real size measured in inch and square inch. No conversion is required in this case, however, due to the nature of the Scale Algorithm.

After the measurement is completed and size of wound is calculated, the app will unwind from the Take Picture scene to Wound Info scene, and the size is passed to Wound Info scene and stored as one of its property. It can then be displayed in Wound Info page when loadData function is called.

\section{Evaluation}

Market evaluation was performed through expert feedback and end customer acceptance. eKare reviewed the wallet app product, and before accepting the product provided some feedback requiring small changes. eKare experts pointed out that the Scaling Method measures both the height and width of the reference card in the picture, but the user only needs to measure the length of one side of the reference card. Either height or width is required, but not both. Since both the real height and width (in inches) have already been defined in the program, and the program cannot 
tell whether the user measures height or width in the real-time measurement process, the program will assume the user always measures the width of the reference card. The user is notified of this setting in a pop-up window shown before the measurement starts. In this way the measurement process is further simplified, as only 6 points instead of 8 points need to be marked down.

Throughout the entire development process, extensive testing was performed to ensure the app always met the requirement in all aspects including UI design, input value validation (boundary value and non-null value check), navigation design, database management and data validation and most importantly, the Scaling Method.

Finally, as discussed in section 3, the application of digital planimetry in the mobile environment using this platform was recently validated in a clinical study [13].

\section{Discussion and Future Work}

Currently only a 2D approximate measurement is available in this version. It would be beneficial if the app can incorporate eKare's own computer vision algorithm, which is far more powerful. The other alternative is to upgrade the current Scaling Algorithm by importing image processing library such as OpenCV [16] to replace finger tap measurement. As such libraries can provide out-of-the-box advanced functions capable of doing edge and border detection so that a much more accurate wound area can be calculated.

Another important direction is to switch the eKare wound-assess app from "offline" to "online mode". This requires a lot more effort because the entire system structure would need to be changed. Components of the system that would be affected include authentication, middle-layer API construction, data format requirement, and most importantly the integration of a medical records platform such as openEMR.

In conclusion, the wallet app concept is intriguing as it applies the concept of the free, lightweight "community edition" version of the software, as one might see in open source products [17], to the problem of creating interest and a potential adopter base to upsell to a more complex and commercial system. We are unaware of other applications of this model in the mHealth space, though it is likely some app on one of the app stores provides a "lite" version as this is fairly pervasive in app distribution strategies. However, the key computer vision based features create real technical challenges to producing a feature-rich app on a reduced platform. This is a significant difference compared to the motivations and requirements of typical free editions on app stores, which are usually motivated by an alternate revenue model (in-app advertisements). While we are not yet at the point where we can collect evidence this approach will bear fruit, it will be interesting to see if this "wallet app" motivation appears in app markets in the near future.

\section{References}

[1] Jørgensen, L.B., Jens A.S., Jemec, G.BE, and Yderstrædem K.B. "Methods to assess area and volume of wounds-a systematic review." International Wound Journal (2015).

[2] Khoo, R., and Jansen, S. "The Evolving Field of Wound Measurement Techniques: A Literature Review." Wounds: a compendium of clinical research and practice 28 , no. 6 (2016): 175.

[3] Sen, C., Gordilo, G., Roy, S. et al. Human skin wounds: a major and snowballing threat to public health and the economy. Wound Repair Regen. 2009; 17:6, 763-771.

[4] Gethin G. The Importance of Continuous Wound Measuring. Wounds. 2006. 2:60-68.

[5] Shah, Aj, C. Wollak, and J. B. Shah. "Wound

Measurement Techniques: Comparing the Use of Ruler Method, 2D Imaging and 3D Scanner." Journal of the American College of Clinical Wound Specialists 5.3 (2013): 52-57.

[6] Sugama J., Matsui Y., Sanada H., Konya C., Okuwa M., and Kitagawa A. A study of the efficiency and convenience of an advanced portable Wound Measurement System (VISITRAK). Journal of clinical nursing, vol. 16(7), 2007, pp. 1265-9.

[8] Flanagan M., Improving accuracy of wound measurement in clinical practice. Ostomy/wound management, vol. 49(10), 2003 , pp. 28-40.

[9] Little C., McDonald J., Jenkins M.G., McCarron P., An overview of techniques used to measure wound area and volume. Journal of wound care, vol.18(6), 2009, pp. 250-3.

[10] Williams C., Wound-measuring methods. Community nurse, vol. 3(8), 1997, pp46-8.

[11] Betz C., Using a programmable pneumatic device with truncal therapy to facilitate wound healing: a case series. Ostomy/wound management, vol. 55(3):34-6, 2009, pp. 3840.

[12] Plassmann P., Melhuish J.M., Harding K.G., Methods of measuring wound size: a comparative study. Ostomy/wound management, vol. 40(7):50-2, 1994, pp. 5660.

[13] Anghel, E.L., Kumar, A., Bigham, T.E., Maselli, K.M., Steinberg, J.S., Evans, K.K, Kim, P.J., and Attinger, C.E., "The Reliability of a Novel Mobile 3-dimensioal Wound Measurement Device", Wounds Epub, August 15, 2016.

[13] Mayrovitz H.N., Soontupe L.B., Wound areas by computerized planimetry of digital images: accuracy and reliability. Advances in skin \& wound care, vol. 22(5):222-9, 2009.

[14] Blake, M.B., Cleary, K., Ranjan, S., Ibanez, L., and Gary, K. "Use Case Driven Component Specification: A Medical Applications Perspective to Product Line Development" ACM Symposium for Applied Computing, Santa Fe, NM, March 2005. 
[15] Patwardhan, M, Stoll, R., Hamel, D.B., Amresh, A., Gary, K.A. and Pina, A., "Design a Mobile Application to Support the Indicated Prevention and Early Intervention of Childhood Anxiety", Proceedings of the National Institutes of Health Conference on Wireless Health (WH'15), Bethesda, MD, October 2015.

[16] Bradski, G., \& Kaehler, A. Learning OpenCV:

Computer vision with the OpenCV library. O'Reilly Media, Inc. 2008.

[17] Gary, K., Koehnemann, H., Blakley, J., Goar, C., Mann, $H$., and Kagan, A. "A Case Study: Open Source Community and the Commercial Enterprise", The IEEE $6^{\text {th }}$ International Conference on Information Technology: New Generations, Las Vegas, NV, April 2009. 